\title{
Article \\ Carbonized Cotton Fabric-Based Flexible Capacitive Pressure Sensor Using a Porous Dielectric Layer with Tilted Air Gaps
}

\author{
Yelin Ko, Chi Cuong Vu and Jooyong Kim * \\ Department of Organic Materials and Fiber Engineering, Soongsil University, Seoul 06978, Korea; \\ yelinko@ssu.ac.kr (Y.K.); vuchicuong@soongsil.ac.kr (C.C.V.) \\ * Correspondence: jykim@ssu.ac.kr; Tel.: +82-2-820-0631
}

Citation: Ko, Y.; Vu, C.C.; Kim, J. Carbonized Cotton Fabric-Based Flexible Capacitive Pressure Sensor Using a Porous Dielectric Layer with Tilted Air Gaps. Sensors 2021, 21, 3895. https://doi.org/10.3390/s21113895

Academic Editor: Marc Ramuz

Received: 7 May 2021

Accepted: 2 June 2021

Published: 4 June 2021

Publisher's Note: MDPI stays neutral with regard to jurisdictional claims in published maps and institutional affiliations.

Copyright: (c) 2021 by the authors. Licensee MDPI, Basel, Switzerland. This article is an open access article distributed under the terms and conditions of the Creative Commons Attribution (CC BY) license (https:// creativecommons.org/licenses/by/ $4.0 /)$.

\begin{abstract}
Flexible and wearable pressure sensors have attracted significant attention owing to their roles in healthcare monitoring and human-machine interfaces. In this study, we introduce a widerange, highly sensitive, stable, reversible, and biocompatible pressure sensor based on a porous Ecoflex with tilted air-gap-structured and carbonized cotton fabric (CCF) electrodes. The knitted structure of electrodes demonstrated the effectiveness of the proposed sensor in enhancing the pressure-sensing performance in comparison to a woven structure due to the inherent properties of naturally generated space. In addition, the presence of tilted air gaps in the porous elastomer provided high deformability, thereby significantly improving the sensor sensitivity compared to other dielectric structures that have no or vertical air gaps. The combination of knitted CCF electrodes and the porous dielectric with tilted air gaps achieved a sensitivity of $24.5 \times 10^{-3} \mathrm{kPa}^{-1}$ at $100 \mathrm{kPa}$, along with a wide detection range $(1 \mathrm{MPa})$. It is also noteworthy that this novel method is lowcost, facile, scalable, and ecofriendly. Finally, the proposed sensor integrated into a smart glove detected human motions of grasping water cups, thus demonstrating its potential applications in wearable electronics.
\end{abstract}

Keywords: capacitive pressure sensor; carbonized cotton fabric (CCF); carbonization; porous dielectric layer; particle-template method; tilted air gaps

\section{Introduction}

Flexible pressure sensors have received tremendous attention due to their potential in monitoring human health [1-3], human-machine interaction systems [4,5], and intelligent robotics [6-8]. Thus far, numerous strategies have been proposed to develop pressure sensors with excellent sensing performance based on various sensing mechanisms, including piezoresistive [9,10], capacitive [11-14], piezoelectric [15,16], and triboelectric effects [17]. Among these approaches, a capacitive-type pressure sensor has attracted the most interest owing to its fast response, high reversibility, temperature insensitivity, simple structure and fabrication process, and low power consumption [18,19]. In general, a capacitive pressure sensor consists of two parallel electrodes and a dielectric layer placed between them. When pressure is applied to a capacitive-type sensor, the thickness of the dielectric decreases, thus changing the capacitance variation in the sensor.

However, capacitive pressure sensors typically exhibit low sensitivities due to the relatively small changes in the capacitance of parallel plates [20]. Therefore, several methodologies have been developed to tune the performance of capacitive pressure sensors, which are mostly determined by the deformability of the dielectric layer. One of the most popular efforts to modify the dielectric layer for sensitivity enhancement involves engineering microstructures on the elastomer surface. Microstructured patterns, such as convex [21], pyramids [22], pillars [23], and waves [24], have been proved to be effective in yielding ultrahigh sensitivity. However, the working range is typically limited to $<10 \mathrm{kPa}$ because the patterns quickly collapse even when subjected to low pressure, thereby making 
the strategy less desirable for most application settings [1]. In addition, this method requires time-consuming, expensive, and complicated process steps to fabricate the microstructure silicone mold.

Another approach that focuses on the formation of porous structures inside the dielectric layer has been proposed as an alternative to the microstructuring of elastomers, including various fabrication techniques such as the particle-template method $[1,11,25,26]$, chemical foaming method [27], and emulsion-template method [28]. Among these, the particletemplate method has emerged as a promising strategy with great potential for providing higher deformability to resulting sensors, along with ease of fabrication at low cost. In this strategy, easily dissolvable particles (sugar or $\mathrm{NaCl}$ ) are added to uncured silicone rubber, such as polydimethylsiloxane (PDMS) $[1,11]$ or Ecoflex $[25,26]$. After the silicone elastomers are cured, the residual particles are dissolved and removed to obtain a porous dielectric layer. In addition, this method can offer a wide dynamic working range to resulting sensors. In general, it has been known that typical tactile pressures are distributed in low-pressure regimes (gentle touch, $0-10 \mathrm{kPa}$ ) and medium-pressure regimes (object manipulation, $10-100 \mathrm{kPa})[20,29]$. Therefore, in order to monitor human motions, a pressure sensor needs to be sensitive over a working range up to $100 \mathrm{kPa}$ [26] to cover the overall tactile pressures. It has been frequently reported that pressure sensors fabricated by the particletemplate strategy can work at the low-pressure $(0-10 \mathrm{kPa})$ [25] and medium-pressure regimes $(10-100 \mathrm{kPa})[11,12]$ and broader ranges $(130 \mathrm{kPa}[26], 400 \mathrm{kPa}[1])$.

The structured pores inside elastomers have been demonstrated to be effective in improving the sensitivity of capacitive pressure sensors by reducing the stiffness of elastomers and increasing the effective dielectric constant due to the gradual closure of pores. For example, Hwang et al. [1] demonstrated that a hierarchically structured porous PDMS-based pressure sensor exhibits 22.5 times greater sensitivity $\left(1.8 \times 10^{-1} \mathrm{kPa}^{-1}\right)$ than a bulk PDMSbased sensor. Atalay et al. [11] proposed a pressure sensor using porous PDMS and textile electrodes with an improved sensitivity of $1.21 \times 10^{-2} \mathrm{kPa}^{-1}$. Kwon et al. [26] fabricated a sensor with porous Ecoflex and showed that the sensitivity of the resulting sensor was 37.6 times higher than that of a sensor with a bulk dielectric structure. However, the microporous structure still experiences sensitivity saturation in high-pressure regions [14,30]. In addition, the high porosity of the dielectric layer may affect the mechanical stability of the sensor [31] and generate more noise.

Meanwhile, the design of electrodes of a capacitive pressure sensor has been less considered owing to its less apparent relationships with sensitivity. Electrodes are mainly fabricated by mixing conductive nanomaterials, such as Ag nanowires [13,19], graphene [18], and carbon nanotubes [12,30], with flexible substrates or polymer matrices [21]. These nanomaterials are widely known to possess excellent mechanical and electrical properties. However, contact with these materials may pose health risks to manufacturers and users, which should not be neglected for use in human health monitoring. In addition, they require complicated fabrication processes and expensive raw materials. Carbonized cotton fabric (CCF), which is converted from cotton fabrics by a simple thermal treatment, can be a potential candidate to compensate for these drawbacks. The high-temperature carbonization process in low oxygen concentration leaves a carbon skeleton formed from cellulose fibers, and the biomass-derived carbon-based materials exhibit high electrical conductivity and flexibility [32]. Furthermore, the carbonization process is scalable, low-cost, and ecofriendly.

Despite their potential, to the best of our knowledge, CCF has rarely been used as an electrode in capacitive pressure sensors even though some studies have highlighted its application to supercapacitor electrodes [33], strain sensors [34,35], and resistive-type pressure sensors [32,36]. With efforts to enhance the sensitivity by engineering the dielectric layer, a CCF-based capacitive pressure sensor may be widely utilized in a variety of applications taking advantages of its less hazard to the human body and general benefits of a capacitive-type sensor. Herein, we propose a facile and inexpensive yet effective strategy to fabricate a capacitive pressure sensor based on CCF electrodes and a porous Ecoflex as the 
dielectric layer. We optimized the sensor performance in two steps: (1) exploring the effects of textile structures (woven vs. knitted) of CCF on sensitivity and (2) structuring the porous elastomer by introducing air gaps (vertical vs. tilted) to generate additional voids inside. Our sensor, consisting of a porous Ecoflex with tilted air gaps and knitted CCF electrodes, exhibited high sensitivity, which can be attributed to the enhanced deformability of the dielectric and the rough and bulky nature of the knitted structure of electrodes. In addition, the sensor showed delayed pressure-sensing saturation, wide working range, low hysteresis, and high reversibility and durability. As a practical demonstration, we integrated the proposed sensor into a smart glove to monitor human motions during object grasping.

\section{Materials and Methods}

To prepare the electrodes of the capacitive pressure sensor, common 100\% cotton fabrics (woven: $181.3 \mathrm{~g} \cdot \mathrm{m}^{-2}, 0.31 \mathrm{~mm}$ in thickness; knitted: $154.8 \mathrm{~g} \cdot \mathrm{m}^{-2}, 0.32 \mathrm{~mm}$ in thickness) (Figure 1a) were put in an aluminum container and placed in a furnace (B253DK, Hanwon, Korea) for subsequent carbonization treatment. The carbonization process of these pristine cotton fabrics lasted for $1 \mathrm{~h}$ after reaching $800{ }^{\circ} \mathrm{C}$, starting from room temperature. After naturally cooling to room temperature, the CCF (Figure 1b) was rinsed several times with distilled water and completely dried to remove impurities and ashes from its surface. Since CCF is too fragile to be used in its original state, we used thermoplastic polyurethane (TPU) as a supporting substrate, similar to previous studies [32,37] to improve the mechanical strength. A $7 \mathrm{wt} \%$ TPU solution was obtained by dissolving TPU in N,Ndimethylformamide (DMF) with magnetic stirring at $50{ }^{\circ} \mathrm{C}$ and $200 \mathrm{rpm}$ for $6 \mathrm{~h}$. To prepare the CCF/TPU composite with moderate flexibility and strength (Figure 1c), the CCF was dipped in the TPU solution for $5 \mathrm{~min}$ and dried at $80^{\circ} \mathrm{C}$ for $5 \mathrm{~min}$; this process was repeated three times before the final thermal curing at $80{ }^{\circ} \mathrm{C}$ for $120 \mathrm{~min}$. Finally, the CCF/TPU composite material was cut into a size of $1.4 \mathrm{~cm} \times 1.4 \mathrm{~cm}$ with a small extra tip for wire connection $(0.6 \mathrm{~cm} \times 0.2 \mathrm{~cm})$ using a laser cutting machine.

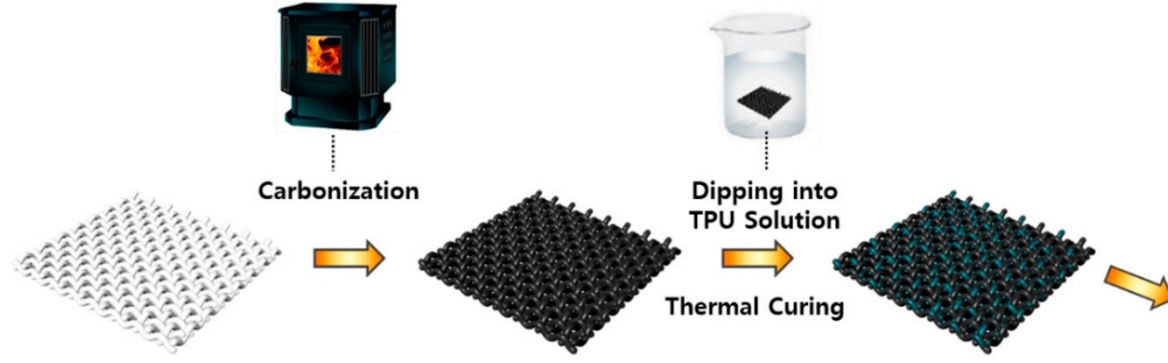

(a) Pristine Cotton Fabric

(b) Carbonized Cotton Fabric (CCF)

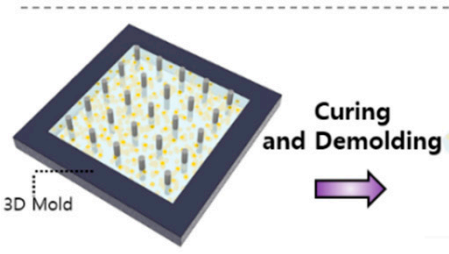

(d) Casting Ecoflex with Sugar Particles

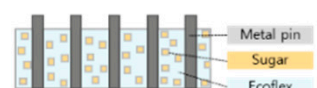

(c) CCF/TPU Composite
Sugar Dissolving and Drying

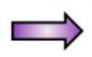

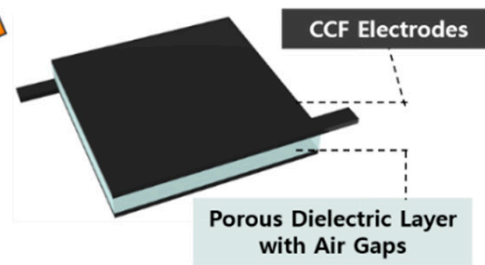

(g) CCF-based

Capacitive Pressure Sensor

Figure 1. Manufacturing process of carbonized cotton fabric (CCF)-based capacitive pressure sensors: (a-c) fabrication of CCF electrodes; (d-f) fabrication of a porous dielectric layer with air gaps; $(\mathbf{g})$ developed capacitive pressure sensor using CCF electrodes and a porous dielectric layer with air gaps. 
A porous dielectric layer with air gaps was prepared by casting Ecoflex with sugar particles (Figure 1d). The Ecoflex (Shore 00-30 hardness, Smooth-on Inc., Macungie, PA, USA) prepolymer solution was obtained by mixing a base (Part A) and cured agent (Part B) at a weight ratio of 1:1. Commercial granulated brown sugar was integrated into the solution at a ratio of 1:2.5 by weight. The Ecoflex solution with sugar granules was poured into a 3D mold with a length, width, and height of $1.4,1.4$, and $0.4 \mathrm{~cm}$, respectively. With a $5 \times 5$ array of metal pins $(0.5 \mathrm{~mm}$ length $\times 0.5 \mathrm{~mm}$ width per pin) inserted into the solution, it was instantly cured at $150{ }^{\circ} \mathrm{C}$ for $3 \mathrm{~min}$ to prevent layer separation in Ecoflex and sugar particles. When structuring tilted air gaps in Ecoflex, metal pins were inserted at a tilting angle of $60^{\circ}$. Subsequently, the solution was placed in an oven at $80{ }^{\circ} \mathrm{C}$ for $2 \mathrm{~h}$ for further thermal curing. After the pins were removed, the cured Ecoflex with sugar particles was removed from the 3D mold (Figure 1e). A porous dielectric elastomer with air gaps was prepared (Figure 1f) after dissolving the sugar particles in distilled water with magnetic stirring at $70{ }^{\circ} \mathrm{C}$ and $120 \mathrm{rpm}$ for at least $24 \mathrm{~h}$. Finally, the capacitive pressure sensor was obtained by sandwiching the air-gapped porous dielectric layer between the CCF electrodes (Figure 1g). To minimize any adverse effects of additional adhesive layers on the performance of the sensor, we hand-sewed the three layers using a common needle and thread.

\section{Results and Discussion}

\subsection{Structures of the Sensor}

The surface morphology of the CCF-based capacitive pressure sensor was obtained by scanning electron microscopy (SEM) (GeminiSEM 300, Carl Zeiss, Oberkochen, Germany). The proposed sensor consists of an air-gapped porous dielectric layer sandwiched between flexible CCF electrodes. In this study, the woven and knitted textile structures of electrodes were first compared to optimize the sensing performance (Figure 2a-d). The fibers in carbonized fabric became finer than those in pristine cotton fabric, indicating that carbonbased materials remained after the carbonization process. The inherent structures of the woven (Figure 2a,b) and knitted structures (Figure 2c,d) were retained in carbonized fabric. Figure $2 \mathrm{e}, \mathrm{f}$ presents the porous structure of the dielectric layer formed by mixing the Ecoflex solution with sugar particles. Figure $2 \mathrm{~g}$, h shows the air gaps formed by metal pins that created additional voids during thermal curing. It is evident from cross-sectional images that vertical (Figure 2i) and tilted air gaps (Figure 2j) were clearly observed. Figure 2k shows the real images of the capacitive pressure sensor with the CCF electrodes and porous dielectric with air gaps. As presented in Figure 2l, the Ecoflex dielectric layer was highly stretchable.

\subsection{Sensing Mechanism}

Figure 3 describes the working principle of the proposed capacitive sensor based on a porous dielectric layer with air gaps and CCF electrodes. In general, the capacitance of a capacitive pressure sensor $\left(\mathrm{C}_{\text {Sensor }}\right)$ (Figure 3a) can be calculated as follows (Equation (1)):

$$
\mathrm{C}_{\text {Sensor }}=\varepsilon_{0} \varepsilon_{r} \frac{A}{d_{0}}
$$

where $\varepsilon_{0}$ is the dielectric permittivity of vacuum, $\varepsilon_{r}$ is the dielectric permittivity of dielectric material, A represents the area of overlapped electrodes, and $d_{0}$ represents the thickness of the dielectric layer. Therefore, the capacitance variation in a sensor under applied pressure is determined by the changes in thickness and the relative permittivity of the porous Ecoflex.

Figure $3 \mathrm{~b}$ presents CCF-based capacitive pressure sensors in the initial and loading states under the same pressure level. When pressure is applied to a pressure sensor with a bulk dielectric without pores, it experiences the barreling phenomenon [26]. As the dielectric constant of the bulk Ecoflex $\left(\varepsilon_{\text {bulk }}\right)$ remains the same under compression, the capacitance variation in the sensor largely depends on the changes in distance between the electrodes $\left(\Delta \mathrm{d}_{\text {bulk }}\right)$. Meanwhile, the pores integrated into a solid silicone elastomer can 
have two benefits, which can significantly improve the sensitivity of CCF-based pressure sensors. First, the porous dielectric is subjected to larger deformation compared to the bulk dielectric under the same level of pressure owing to numerous pores. This leads to greater changes in the thickness of the porous Ecoflex $\left(\Delta \mathrm{d}_{\text {porous }}\right)$, which results in greater variations in the capacitance responses of the sensor.
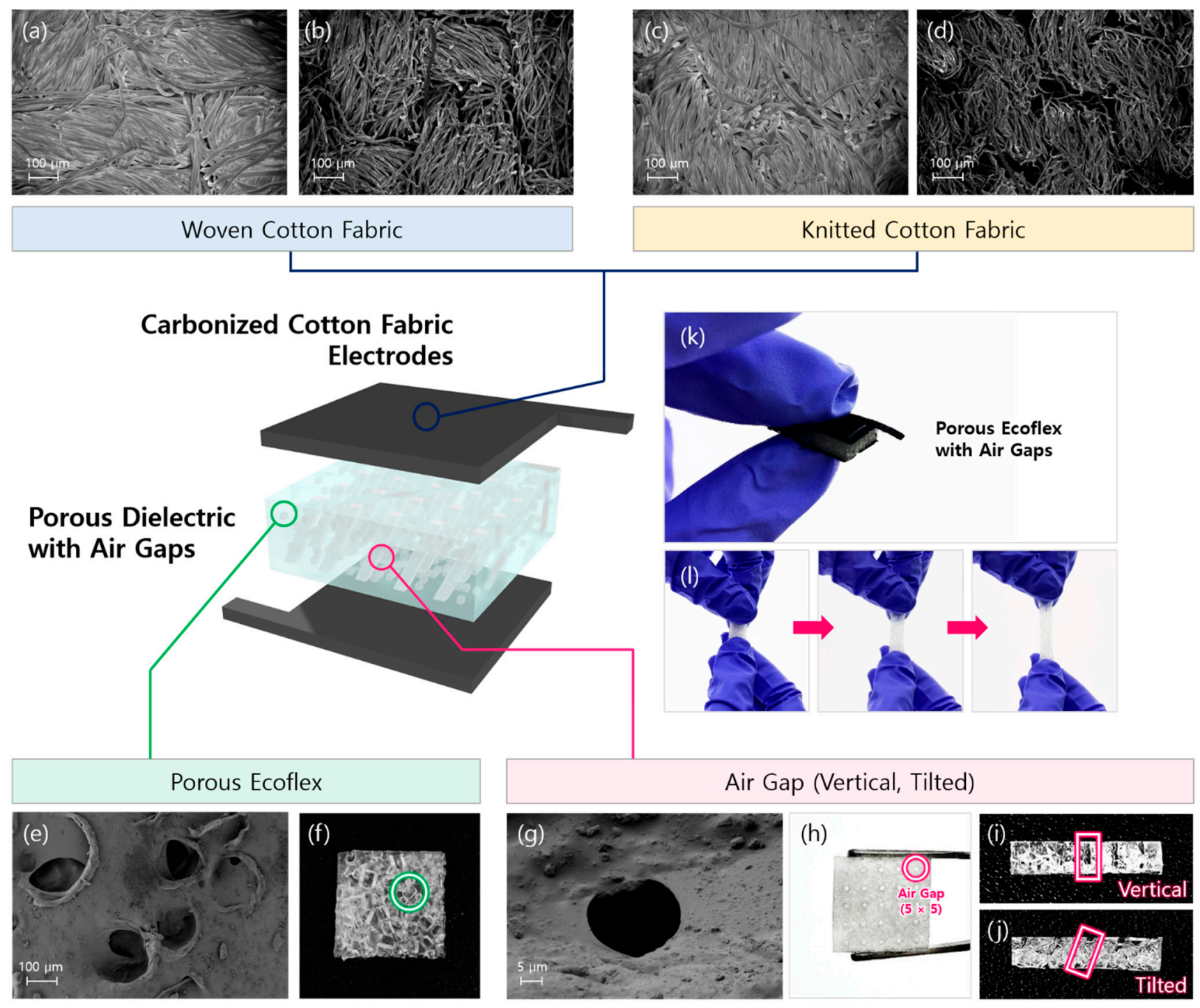

Figure 2. Structure of the CCF-based capacitive pressure sensor and SEM images of the electrodes and dielectric layer. (a) Woven pristine cotton fabric; (b) woven CCF; (c) knitted pristine cotton fabric; (d) knitted CCF; (e) porous structure of Ecoflex as a dielectric layer; (f) top-view image of the porous Ecoflex. The voids inside Ecoflex are the pores created by sugar particles and highlighted in green; $(\mathbf{g}, \mathbf{h})$ air gaps in the porous dielectric; cross-sectional view images of the vertical (i) and tilted air gaps (j) in the porous dielectric. The air gaps were formed by metal pins and highlighted in pink; (k) real image of the CCF-based capacitive pressure sensor with air gaps; (1) high stretchability of the dielectric layer. 


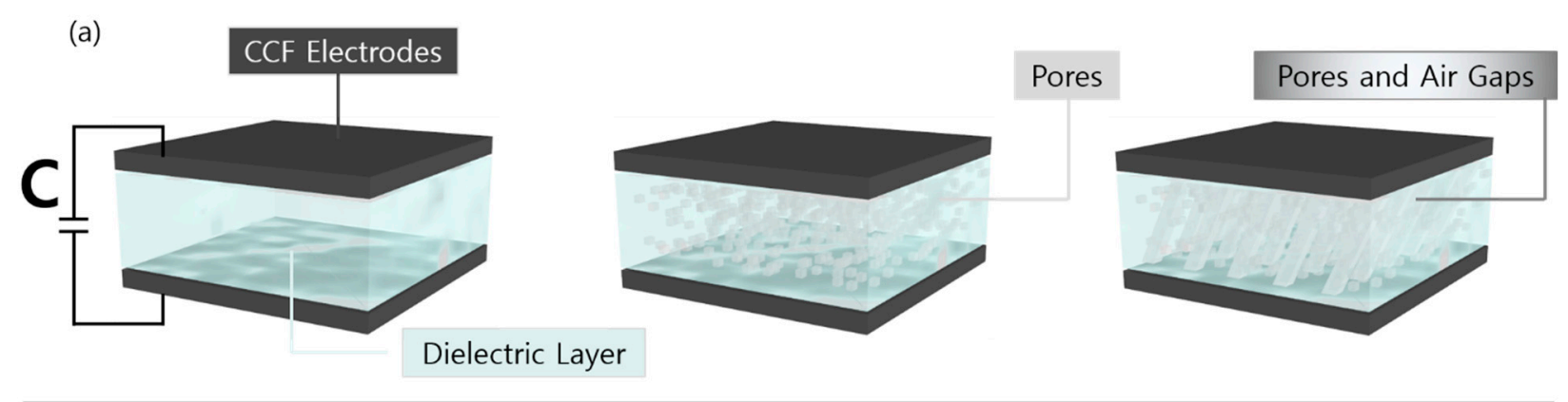

(b)

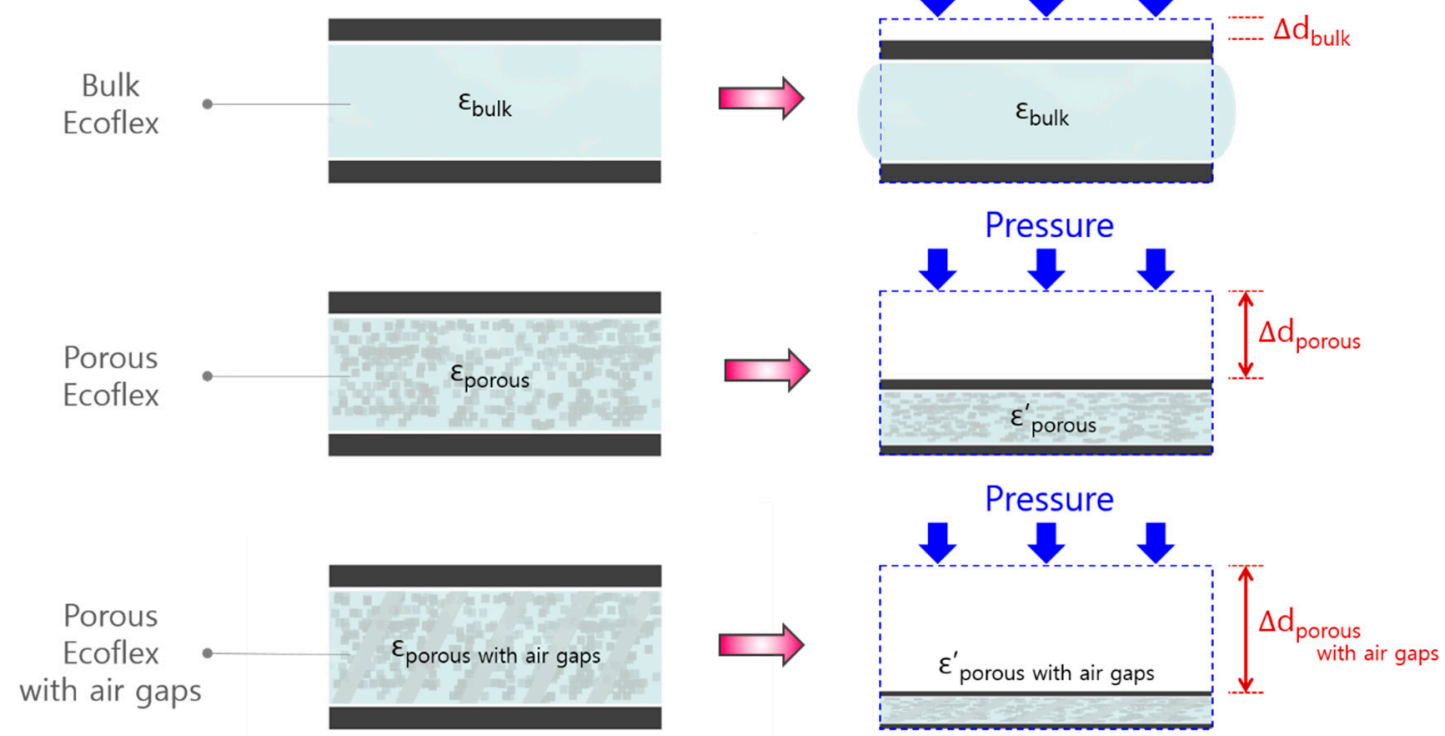

Figure 3. Pressure-sensing mechanisms of capacitive pressure sensors using a bulk Ecoflex, porous Ecoflex, and air gapped-porous Ecoflex. (a) Schematic of sensors with three different structures in the dielectric; (b) schematic of the structural deformation of capacitive pressure sensors using a bulk, porous, air gapped-porous Ecoflex dielectric layer during compressive loading.

Second, the pores under external pressure increase the effective dielectric constant of the porous Ecoflex $\left(\varepsilon_{e}\right)$, which can be described as follows (Equation (2)) [11]:

$$
\varepsilon_{e}=\varepsilon_{\text {air }} V_{\text {air }}+\varepsilon_{\text {Ecoflex }} V_{\text {Ecoflex }}
$$

where $\varepsilon_{\text {air }}=1$ and $\varepsilon_{\text {Ecoflex }}=2.8$ [38], $V_{\text {air }}$ represents the volume fraction of air, and $V_{E c o f l e x}$ represents the volume fraction of pristine Ecoflex. Under compression, the pores in the dielectric layer steadily close, decreasing the volume fraction of air and increasing that of Ecoflex. The lower dielectric constant of pores $\left(\varepsilon_{\text {air }}=1\right)$ is replaced by the higher dielectric constant of silicone elastomer $\left(\varepsilon_{\text {Ecoflex }}=2.8\right)$, which subsequently increases the effective dielectric constant of the porous Ecoflex composite [1]. When air gaps are introduced to this porous elastomer, the sensitivity of the pressure sensor can be further improved owing to enhanced deformability because the gaps will reduce the stiffness of the pristine Ecoflex, creating additional voids inside.

\subsection{Sensitivity Differences between Pressure Sensors Based on Woven and Knitted CCF Electrodes}

We optimized the sensitivity of the CCF-based capacitive pressure sensor with the following two steps: (1) investigation of the effects of textile structures (woven vs. knitted) of CCF electrodes and (2) exploration of the effects of vertical and tilted air-gap structures of the porous dielectric layer. The pressure-sensing performance of the sensor was evaluated using a customized universal testing machine (Dacell Co., Seoul, Korea) and a Keysight 
E4980AL LCR meter (Figure 4a). Figure 4b shows photographs of pristine and carbonized woven and knitted cotton. After the carbonization process at $800{ }^{\circ} \mathrm{C}$, the colors of both pristine woven and knitted cotton changed to black, indicating that the carbon materials were mainly left after the high-temperature pyrolysis. The surface area and weight of the pristine cotton fabrics were reduced by $49.6 \%$ and $89.3 \%$ for the woven structure and $47.2 \%$ and $90.5 \%$ for the knitted structure, respectively. The thickness of both woven and knitted CCF decreased from 0.31 to $0.25 \mathrm{~mm}$ and 0.32 to $0.25 \mathrm{~mm}$, respectively. As shown by the SEM images, naturally formed spaces were observed in the knitted CCF owing to its inherent structure, whereas such space was less present in the woven CCF (Figure 4b).

(a)

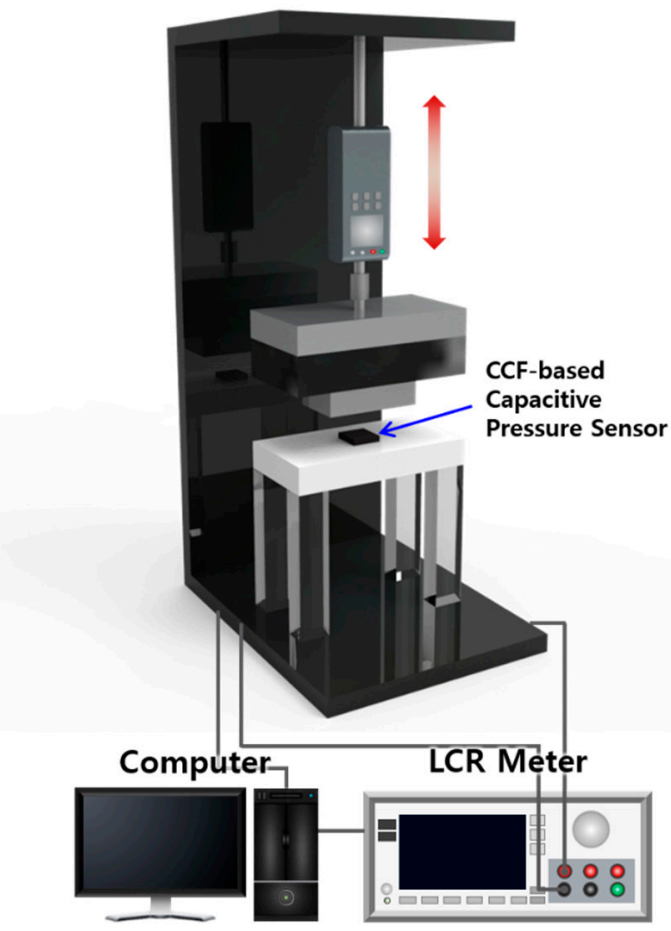

(c)

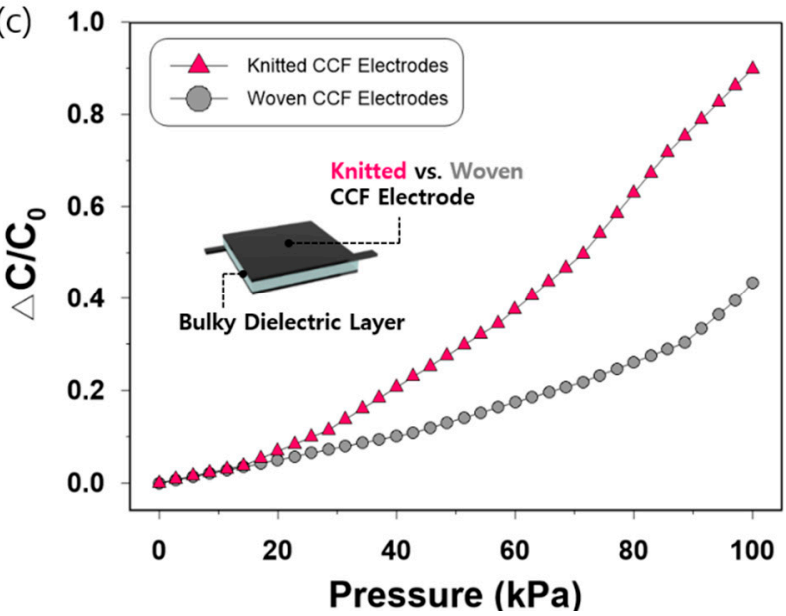

(b)
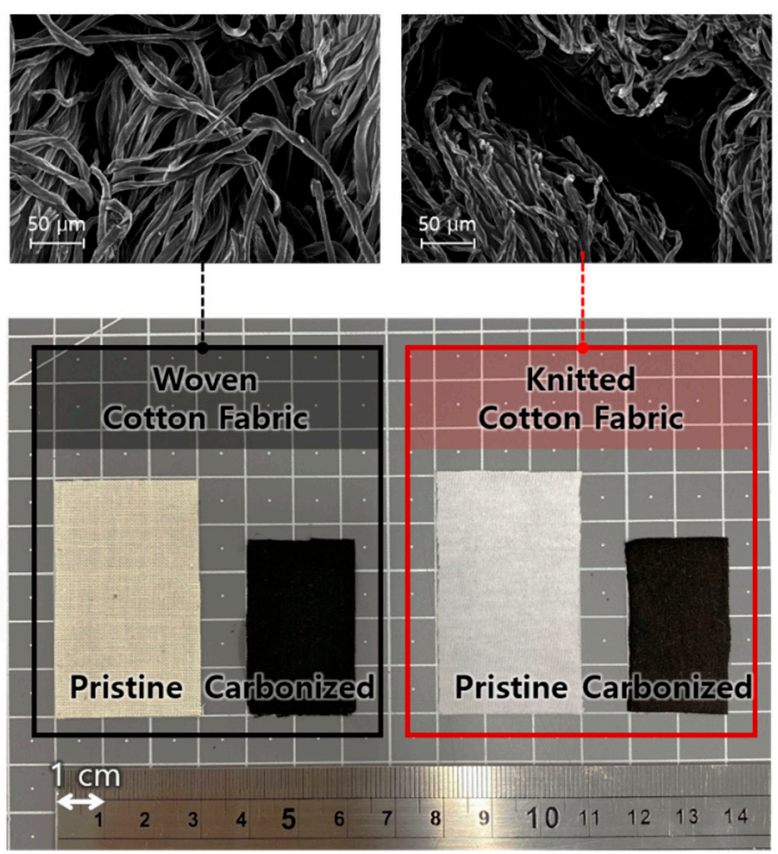

(d)

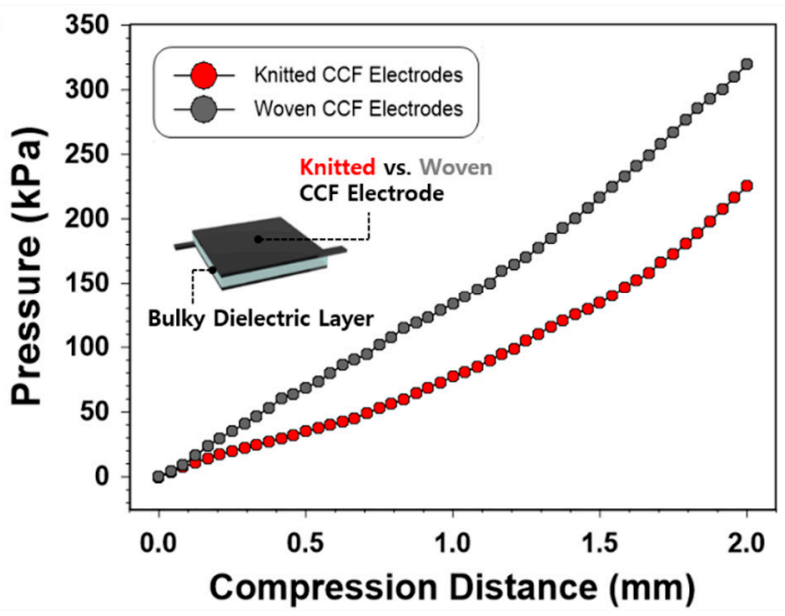

Figure 4. (a) Schematic of the customized universal testing machine; (b) woven and knitted cotton fabrics after carbonization at $800^{\circ} \mathrm{C}$; (c) pressure-response curves of capacitive pressure sensors with knitted and woven CCF electrodes; (d) relationship between the compression distance and applied pressure of sensors with knitted and woven CCF electrodes.

To compare the pressure-sensing sensitivity caused by the two different structures of CCF electrodes, the woven and knitted CCF electrodes were combined with a bulk Ecoflex as a dielectric layer without pores and air gaps. The sensitivity of CCF-based capacitive pressure sensors (S) was calculated as $\mathrm{S}=\triangle \mathrm{C} / \mathrm{C}_{0} / \mathrm{P}$, where $\triangle \mathrm{C}$ and $\mathrm{C}_{0}$ represent the 
change in capacitance and initial capacitance of the sensor, respectively, and P represents the applied pressure. As shown in Figure 4c, the pressure sensor with knitted CCF electrodes exhibited higher sensitivity $\left(9.0 \times 10^{-3} \mathrm{kPa}^{-1}\right)$ than the woven structure $\left(4.4 \times 10^{-3} \mathrm{kPa}^{-1}\right)$ under an applied pressure level of $100 \mathrm{kPa}$. The sensitivity difference between the sensors based on woven and knitted CCF electrodes was first apparent at the pressure threshold of $14.3 \mathrm{kPa}$ (Supplementary Materials Figure S1). The sensitivity of the knitted CCF-based pressure sensors was 1.5, 2, and 2.5 times greater than that of the woven CCF-based sensors at the applied pressure of $25.7 \mathrm{kPa}, 40 \mathrm{kPa}$, and $100 \mathrm{kPa}$, respectively (Figure S1).

As shown in Figure 4d, the compression distance of the pressure sensor based on knitted CCF electrodes was greater than that of woven CCF electrodes under the applied pressure, thus supporting the higher sensitivity of woven CCF-based pressure sensors. Since the same dielectric layers were used, it is highly probable that the difference in pressure sensing performance between the two sensors stems from the inherent properties of the woven and knitted structures. Figure S2 shows that a knitted CCF is more compressible than a woven CCF under a given applied pressure. While a woven fabric is made by interlacing warps and wefts in a perpendicular direction, a knitted fabric is fabricated by interlooping courses and wales. The looping structure of a knitted CCF forms a space between the fabric and bulk Ecoflex, and between the adjacent wales (series of loops running lengthwise in a knitted fabric), making a knitted fabric bulkier than a woven fabric. This naturally formed space between the knitted electrodes and the dielectric is likely to function as a second dielectric layer in the capacitive pressure sensor, thereby inducing higher sensitivity than a woven structure [11].

\subsection{Sensitivity Enhancement by Air-Gap Integration into a Porous Dielectric Layer}

Given the advantages of the knitted structure in inducing higher sensitivity, we utilized knitted CCF electrodes in our sensor when structuring air gaps inside the porous dielectric layer to further enhance the sensitivity. As shown in Figure 5a, our sensor is highly flexible, elastic, and reversible. The air gaps in the porous dielectric layer were generated in two types: vertical and tilted structures (Figure 5b). The tilted air gaps in the structured elastomer demonstrated the highest pressure-sensing performance at an applied pressure of $100 \mathrm{kPa}$ (Figure $5 \mathrm{c}$ ). The sensitivities of pressure sensors with tilted, vertical, and no air gaps in the dielectric layers were $24.5 \times 10^{-3}, 18.8 \times 10^{-3}$, and $13.5 \times 10^{-3} \mathrm{kPa}^{-1}$, respectively. The capacitance variations between the three air-gap structures showed greater differences as the applied pressure increased $(100 \mathrm{kPa})$. The sensitivity of our best sensor based on a porous Ecoflex with tilted air gaps $\left(24.5 \times 10^{-3} \mathrm{kPa}^{-1}\right.$ at $\left.100 \mathrm{kPa}\right)$ was relatively high in comparison to that of sensors with a porous Ecoflex by the particle template method using sugar $\left(12.1 \times 10^{-3} \mathrm{kPa}^{-1}\right)$ [11] or salt $\left(19.9 \times 10^{-3} \mathrm{kPa}^{-1}\right)$ [12].

When a higher pressure of $1000 \mathrm{kPa}$ was applied (Figure $5 \mathrm{~d}$ ), the sensitivity of capacitive pressure sensors with tilted, vertical, and no air gaps in the dielectric decreased to $3.7 \times 10^{-3}, 3.1 \times 10^{-3}$, and $2.3 \times 10^{-3} \mathrm{kPa}^{-1}$, respectively. This is mainly due to the sharp decrease in capacitance changes observed at an applied pressure of $125 \mathrm{kPa}$ (shaded in gray in Figure 5d). The decreasing tendency of sensor sensitivity observed in the three sensors in the higher pressure region (125-1000 $\mathrm{kPa}$ ) can be attributed to the closure of structured pores in the dielectric layer and the elastic resistance of the remaining elastomer, as often addressed in previous research [13]. 
(a)

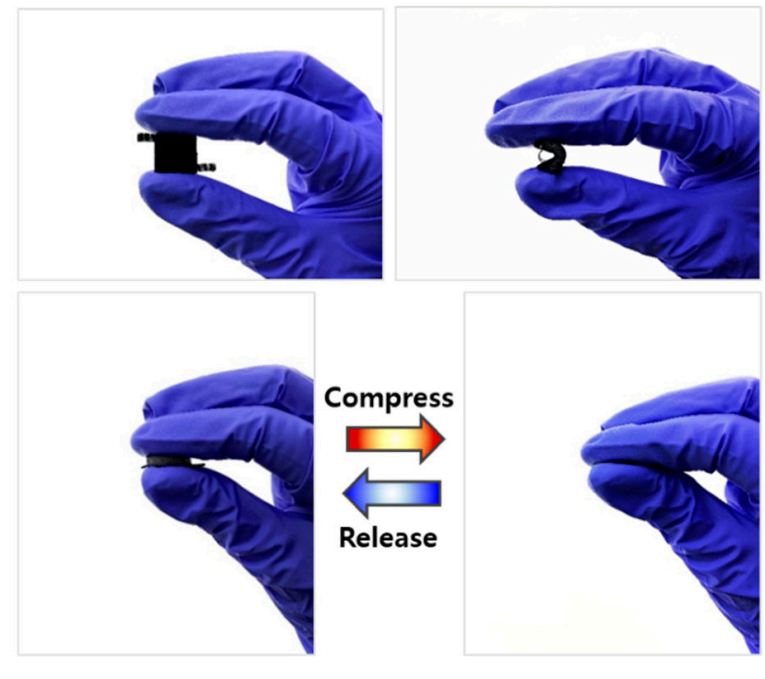

(b)

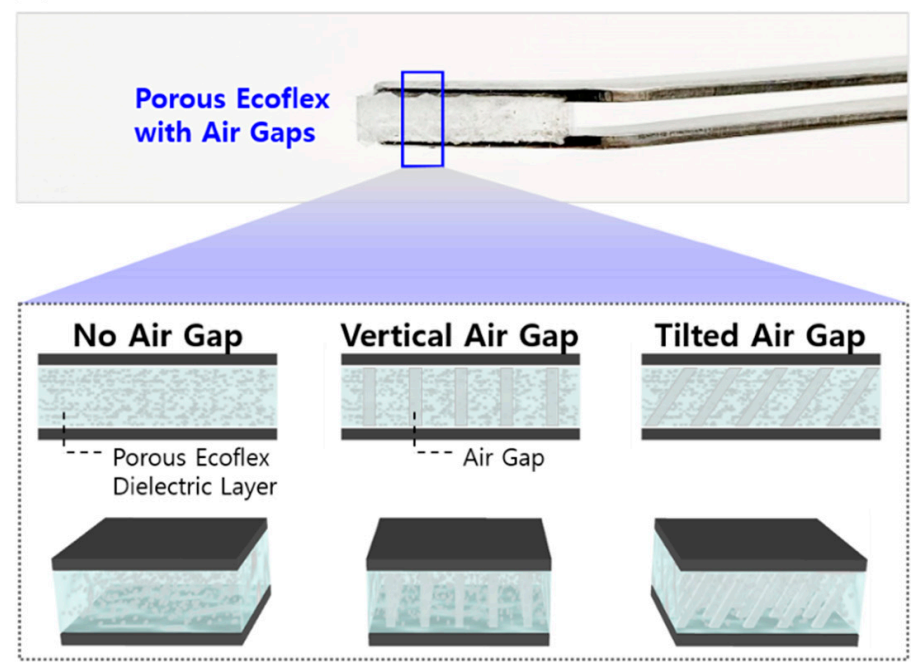

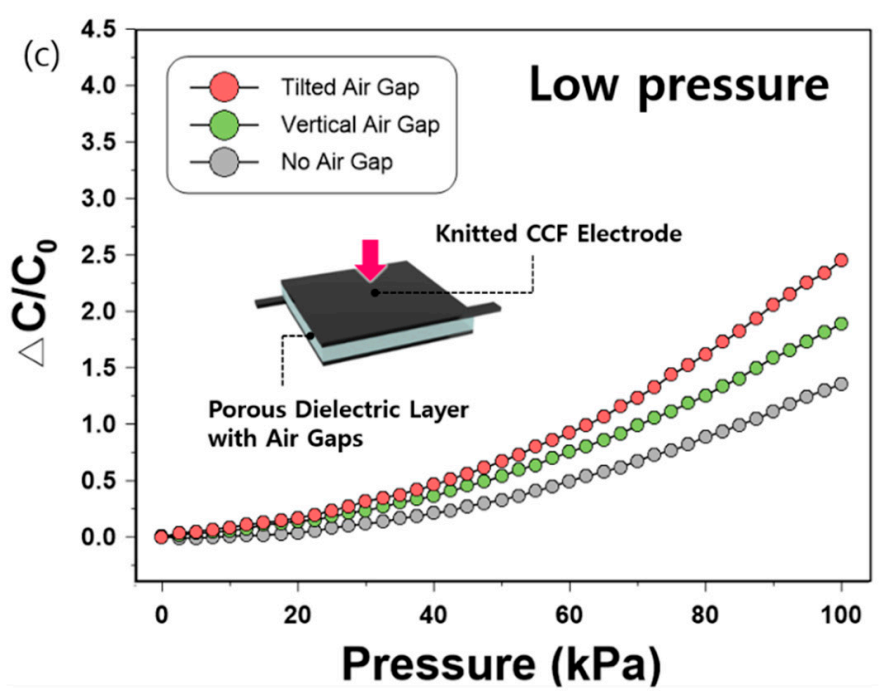

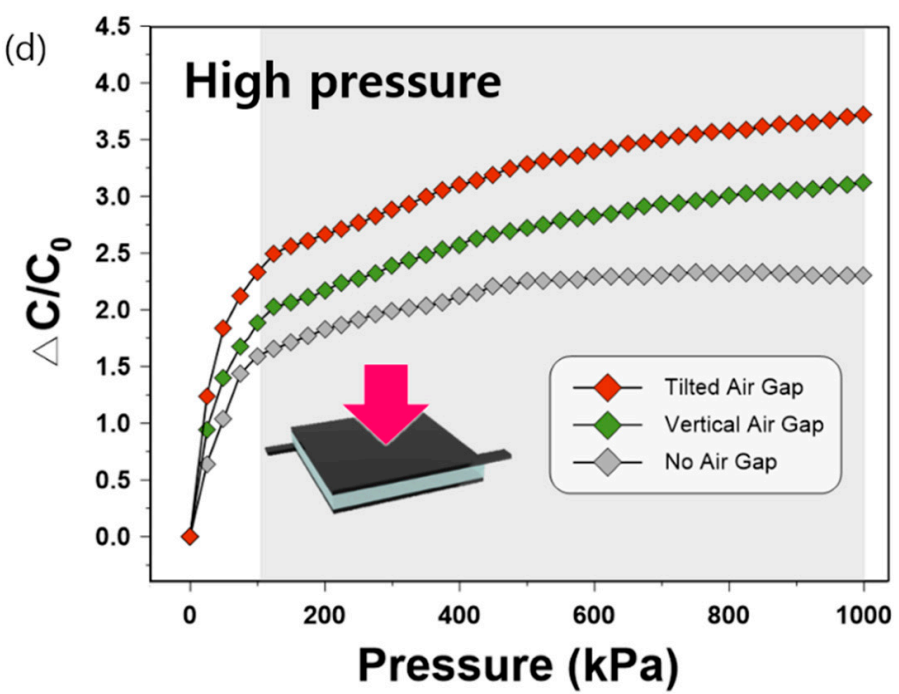

Figure 5. (a) Photographs of the CCF-based capacitive pressure sensor showing the mechanical flexibility and elasticity; (b) air gap structures of the capacitive pressure sensor within the porous dielectric layer; capacitance response of CCF-based pressure sensors based on the porous dielectric layer with no air gaps, vertical air gaps, and tilted air gaps at the applied pressure (c) from 0 to $100 \mathrm{kPa}$ and (d) from 0 to $1000 \mathrm{kPa}$.

Based on the findings in Figure $5 \mathrm{c}, \mathrm{d}$, the following three features of the CCF-based sensor with tilted air gaps in the dielectric can be highlighted: (1) a wide detection range, (2) late saturation of the pressure-sensing performance, and (3) high sensitivity. First, the workable range of our sensor $(0-1000 \mathrm{kPa})$ is outstanding compared to the sensing range of previous research on pressure sensors based on CCF. For example, Chang et al. [32] fabricated a flexible CCF/TPU pressure sensor whose detection range was limited to $0-16 \mathrm{kPa}$ [32]. The multilayer pressure sensor based on CCF/PDMS [36] and the CCF sensor decorated with reduced graphene oxide [2] had a sensing range of 0-200 kPa and 0-500 kPa, respectively. The wide detection range of our sensor can be attributed to the porous structure of the dielectric between the CCF electrodes.

Second, the air gap structure in the dielectric may be advantageous in terms of delaying the pressure-sensing saturation of pressure sensors with a porous dielectric. While the structured pores in the dielectric can lead to wider operating ranges and higher sensitivity in capacitive pressure sensors by increasing the deformability [3,31,39], capacitive pressure sensors with porous dielectrics suffer from rapid saturation of the sensing performance $[14,30]$. It is worth noting that both sensors with an air gap structure (vertical and tilted) exhibited 
a steady increase in capacitance variations, whereas the pressure sensor with no air gap displayed saturated responses after an applied pressure of $500 \mathrm{kPa}$. This may show the advantages of air gaps that penetrate the dielectric layer; the air gaps can still contribute to the changes in the distance between CCF electrodes after the smaller pores become saturated, which may slow down the saturation of the capacitive pressure sensor.

Finally, the sensor with tilted air gaps in the porous Ecoflex exhibited higher sensitivity than that with vertical air gaps in both low-and high-pressure regions. To understand why the tilted air gaps induced higher sensitivity when integrated into the dielectric of a pressure sensor, we compared the dielectric constants of the four different structures of elastomer, namely, bulk Ecoflex and porous Ecoflex with no air gaps, vertical air gaps, and tilted air gaps (Figure 6a). In addition, the compression distances of elastomers under the same applied pressure were explored (Figure 6b). The dielectric constant was measured using a Keysight E4980AL LCR meter with a dielectric material fixture (1020, Wayne Kerr, UK) using a non-contact electrode method at a frequency of $1 \mathrm{kHz}$. As shown in Figure 6a, the dielectric constants of the porous Ecoflex with and without air gaps were lower than those of bulk Ecoflex (no pores, no air gaps) owing to the increased number of voids. Notably, the dielectric constants of the porous Ecoflex with no, vertical, and tilted air gaps did not significantly differ, which indicates that the air gaps in the dielectric may not significantly affect the initial relative permittivity of a material.

(a)

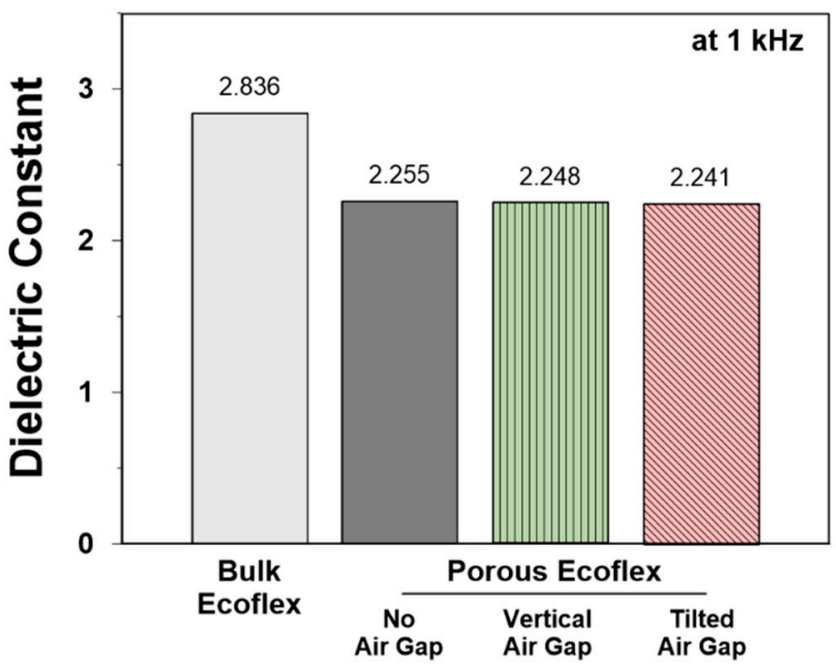

(c)

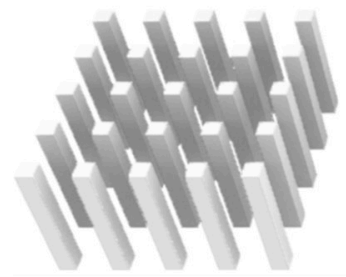

Vertical

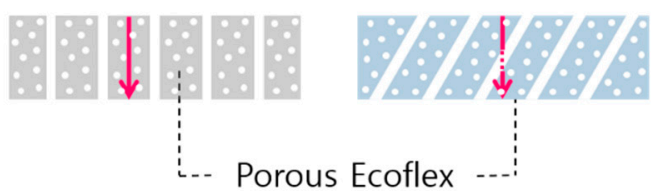

(b)

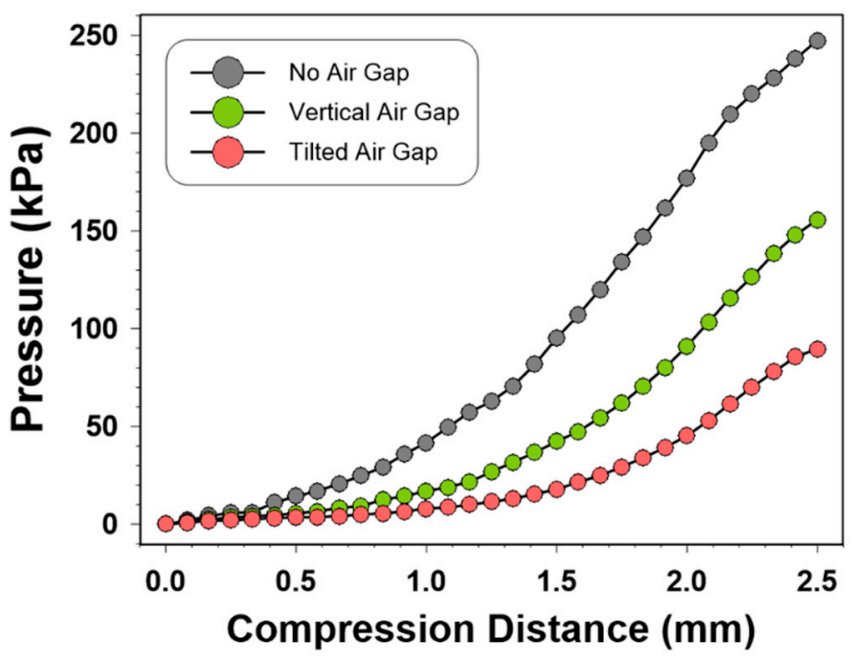

Figure 6. (a) Dielectric constants of the bulk dielectric, porous dielectric with no air gaps, vertical air gaps, and tilted air gaps at a frequency of $1 \mathrm{kHz}$; (b) relationship between the compression distance and applied pressure of CCF-based capacitive pressure sensors with no air gaps, vertical air gaps, and tilted air gaps; (c) schematic of air gaps before and after compression to $30 \%$. 
However, as demonstrated in Figure 6b, the sensor with the porous dielectric with tilted air gaps exhibited the lowest level of applied pressure required to compress the sensor to a given distance. For example, the applied pressure to compress the sensors by $2 \mathrm{~mm}$ for the tilted gap structure was $45 \mathrm{kPa}$, whereas those for the vertical and no air gap structures were 91 and $177 \mathrm{kPa}$, respectively. Therefore, the higher deformability found in the porous Ecoflex with tilted air gaps is likely to result in greater changes in capacitance when compressed, thereby increasing the sensitivity. Figure $6 \mathrm{c}$ presents a schematic of the vertical and tilted air gaps before and after compression. In a vertical structure, air gaps are less likely to affect the compressibility of the adjacent part of the porous Ecoflex. However, the tilted gaps create empty spaces near the remaining Ecoflex; less force is required to compress the tilted structure owing to tilted air gaps that substitute the porous Ecoflex in the direction of pressure. Therefore, the tilted structure of air gaps may be more effective in reducing the stiffness of elastomer than the vertical structure. In addition, as observed in the $70 \%$ compressed state, the tilting angle of $60^{\circ}$ decreases as pressure is applied, which may increase the space occupied by tilted air gaps in the dielectric.

Accordingly, it is probable that the higher sensitivity observed in the tilted air-gap structure was induced by higher deformability, which effectively increased the changes in distances between the electrodes, with less relation to the initial dielectric constants of materials. Here, it should be noted that our investigation does not consider the differences in the effective dielectric constant under compression stemming from structural difference, which deserves further systematic investigation. In addition, the tilting angels of the air gaps in the dielectric layer may significantly affect the pressure-sensing performance, such as sensitivity and working ranges. The optimum of tilting angles, which our study did not explore, should be demonstrated in future research. Furthermore, Luo et al. [23] fabricated a capacitive pressure sensor based on tilted micropillar arrays and attributed the sensitivity enhancement effect of the tilted structure to the bending deformation. They mentioned that bending deformation would facilitate the distance changes between the electrodes compared to compression deformation that traditional vertical arrays undergo. This phenomenon may be applied to the structure of our sensor, which should be further investigated in future research.

The pressure-sensing properties of our best CCF-based capacitive pressure sensor (a porous dielectric layer with tilted air gaps) were characterized (Figure 7). As demonstrated in Figure 7a, our sensor exhibited reversible capacitance variations without notable hysteresis. The degree of hysteresis $(D H)$, which quantitatively indicates the hysteresis performance, was calculated as follows (Equation (3)) [40]:

$$
D H=\frac{A_{\text {Loading }}-A_{\text {Unloading }}}{A_{\text {Loading }}} \times 100(\%)
$$

where $A_{\text {Loading }}$ and $A_{\text {Unloading }}$ are the areas under the loading and unloading curves, respectively. The $D H$ of the proposed CCF-based pressure sensor was $8.8 \%$. This low hysteresis can be attributed to the existence of pores and air gaps, which decreases the volume fraction of Ecoflex. Therefore, the viscoelastic characteristics of Ecoflex are reduced in structured elastomers, inducing negligible hysteresis [41].

When a pressure of $100 \mathrm{kPa}$ was applied, the capacitance of the CCF-based capacitive pressure sensor with tilted air gaps displayed immediate increase with a response time of less than $0.1 \mathrm{~s}$ (Figure $7 \mathrm{~b}$ ). When the pressure was released, the sensor also exhibited rapid recovery time of less than $0.1 \mathrm{~s}$ (Figure 7b). Figure 7c shows the capacitance responses of the sensor under various periodic pressures of 10,50,200, and $500 \mathrm{kPa}$ at $0.1 \mathrm{~Hz}$. The electrical responses of the sensor were highly reversible and stable during the five loading and unloading cycles, which can be attributed to the elastic properties of the Ecoflex dielectric layer. As already shown in Figure $5 c, d$, the signal amplitudes did not necessarily correspond to an increase in the applied pressure. 

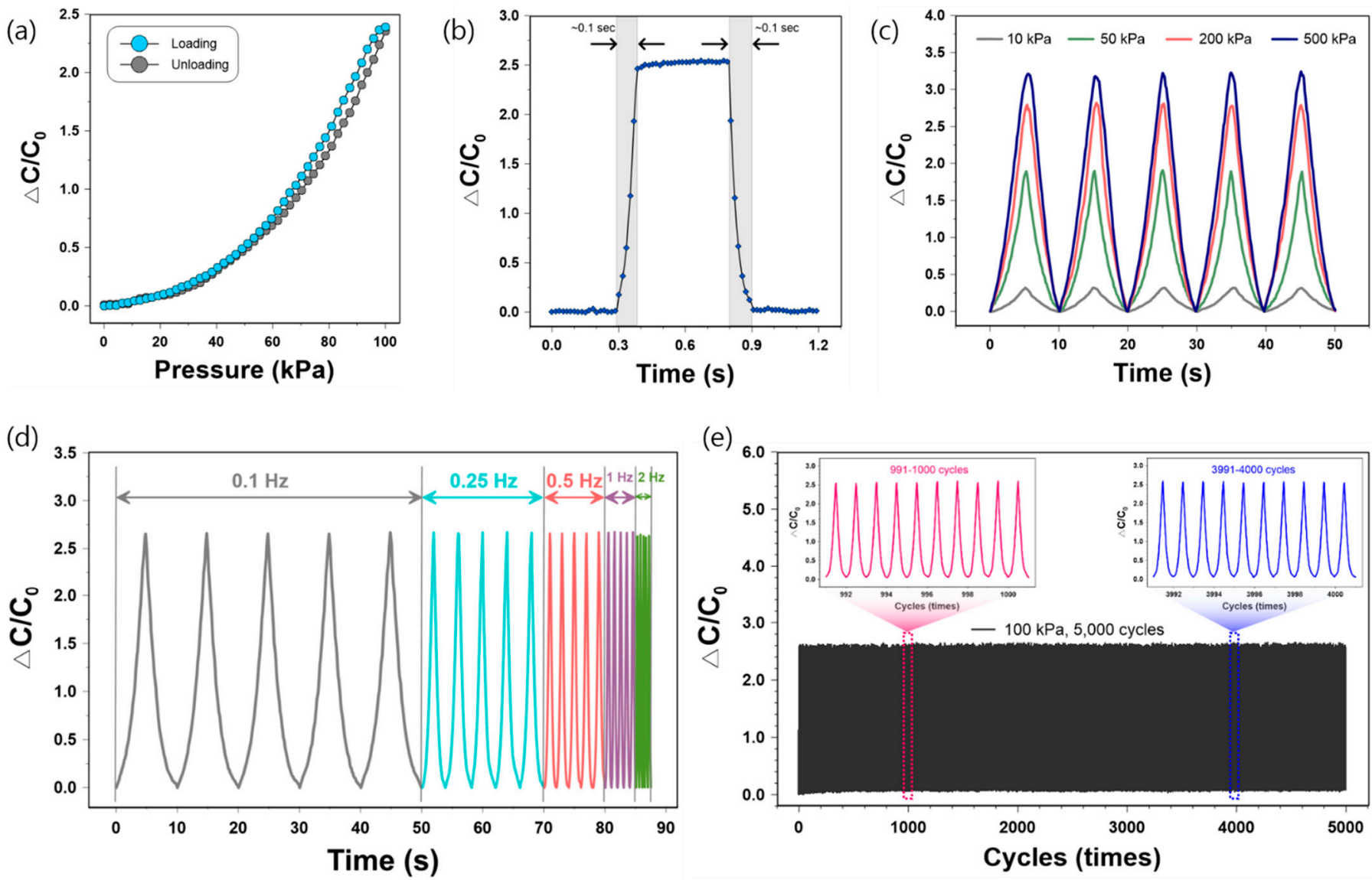

Figure 7. Typical pressure-sensing characteristics. (a) Hysteresis properties of CCF-based capacitive pressure sensors; (b) response and recovery time of pressure sensors; (c) dynamic response of pressure sensors under periodic applied pressures of 10,50,200, and $500 \mathrm{kPa}$; (d) dynamic response of pressure sensors at different frequencies of $0.1,0.25,0.5,1$, and $2 \mathrm{~Hz}$; (e) capacitance response of pressure sensor during 5000 loading and unloading cycles at an applied pressure of $100 \mathrm{kPa}$.

To evaluate the pressure-sensing responses of the pressure sensor at higher frequencies, we tested the sensor performance under an applied pressure of $100 \mathrm{kPa}$ by increasing the frequency from 0.1 to $2 \mathrm{~Hz}$ (Figure 7d). Notably, the sensor signals at different frequencies displayed stable dynamic responses with no apparent changes in amplitude, except for at $2 \mathrm{~Hz}$ where the amplitude showed slight decreases. Durability is of great importance for the long-term practical application of pressure sensors, showing that it can maintain their performance after repeated use. Figure 7e presents the capacitance variations in the CCF-based pressure sensor with tilted air gaps under a pressure of $100 \mathrm{kPa}$ during 5000 loading-unloading cycles. The performance of the pressure sensor was stable after 5000 cycles. This outstanding reproducibility and durability can be ascribed to the highly resilient characteristics of Ecoflex as a dielectric layer.

\subsection{Applications of CCF-Based Capacitive Pressure Sensor in Human-Motion Monitoring}

The wearable applications of the proposed capacitive pressure sensor using a porous Ecoflex with tilted air gaps as the dielectric layer and knitted CCF electrodes were tested through grasping motions. As shown in Figure 8a, we developed a smart glove using the sensor as a portable prototype. Electrical wires (AWG 32) were fixed on the top and bottom sides of the CCF electrode using a thin thermal film by a heat press (ISP0450MR, INNOSTA, Hanam, Korea) at $150{ }^{\circ} \mathrm{C}$ for $15 \mathrm{~s}$ to ensure a firm connection between the wires and electrodes. The sensor was integrated into the index finger of the glove using a doublesided thermal film and the same heat press. The prototype glove with the developed sensor was prepared by connecting it to a hardware platform. The hardware platform included an integrated microcontroller (MCU) with Bluetooth 5.0 (Nrf52840), a lipo-battery 
(3.7 V), and a resistor $(2 \mathrm{M} \Omega)$. The sensing signal was collected every $50 \mathrm{~ms}$ and smoothed using a digital moving average filter (finite impulse response filter). It accepts 10 input samples at a time and takes their average to produce a single output point. The average value is transmitted to a phone, desktop, or tablet monitor using a wireless protocol (Bluetooth low-energy).

(a)
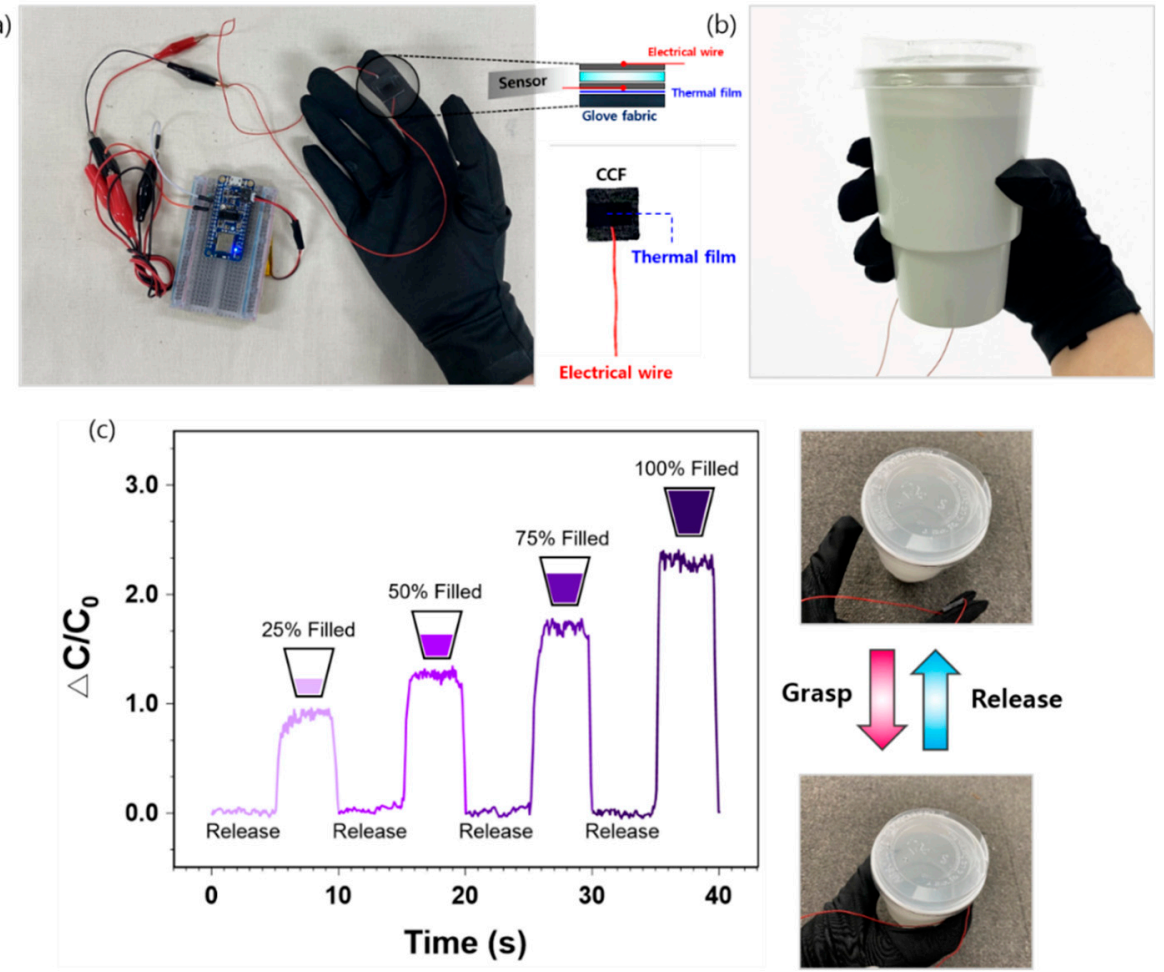

Figure 8. (a) Prototype of the finger-grasping-monitoring glove; (b) water-cup grasping with the smart glove; (c) capacitance variations of grasping motions with a $25 \%, 50 \%, 75 \%$, and $100 \%$ filled water cup.

During the grasping test, subjects were asked to grasp and hold a water cup (200 g) for $10 \mathrm{~s}$ (Figure $8 \mathrm{~b}$ ), followed by a $10 \mathrm{~s}$ release. The sensor signal immediately increased upon grasping the cup and decreased during the release period (Figure 8c). The CCFbased capacitive pressure sensor was sensitive enough to detect the changes in cup weight, which was controlled by pouring $75,150,225$, and $300 \mathrm{~mL}$ water to fill the cup by $25 \%, 50 \%$, $75 \%$, and $100 \%$, respectively. The amplitude showed an apparent increase with the amount of water. This sensitive and repeatable pressure-sensing performance demonstrates the possible applications of the sensor to wearable electronics or smart robotics, where monitoring is required for grasping motions. However, the capacitance variations in the grasping motions showed slight noise during the 5-s grasping and 5-s releasing periods (Table S1), which should be addressed and considered for practical applications. In addition to robotic fingers, our sensor may also be applied to human-motion monitoring such as finger and knee bending, walking, running, and jumping. The scalable fabrication process of the proposed sensor may be particularly beneficial to these applications; the sensor can be readily fabricated by the straightforward process with inexpensive low materials (Ecoflex, sugar, and cotton).

\section{Conclusions}

In conclusion, we presented a novel capacitive pressure sensor with CCF electrodes and an air-gapped porous Ecoflex using an easy, low-cost, and scalable strategy. Commercial cotton fabric was converted into electrodes by thermal treatment at $800{ }^{\circ} \mathrm{C}$, and the air-gapped porous Ecoflex was fabricated using sugar particles and a $5 \times 5$ array of metal 
pins. The combination of knitted CCF electrodes and the porous dielectric with tilted air gaps yielded the highest sensitivity $\left(24.5 \times 10^{-3} \mathrm{kPa}^{-1}\right.$ at $\left.100 \mathrm{kPa}\right)$. This can be attributed to the naturally formed space in knitted fabrics and the effectiveness of the tilted structure in reducing the stiffness of the dielectric, leading to its high deformability. In addition, the proposed sensor exhibited outstanding flexibility, reversibility, durability, low hysteresis, fast response and recovery time, and a wide detection range (1 MPa). Furthermore, because the electrodes of the sensor were derived from cotton, the sensor can be highly biocompatible and ecofriendly. To demonstrate its applications, we developed a prototype smart glove to monitor grasping motions. The sensor was sensitive enough to detect grasping motions under different cup weights. Therefore, we expect that this newly developed sensor with a facile yet effective strategy has great potential in a wide range of applications.

Supplementary Materials: The following are available online at https: / www.mdpi.com/article / 10.3390/s21113895/s1, Figure S1: Sensitivity curves of capacitive pressure sensors with knitted and woven CCF electrodes. The blow arrow indicates the pressure threshold where the sensitivity differences appeared between the knitted and woven CCF-based pressure sensors. The purple dotted lines show the pressure levels where the sensitivity of the knitted CCF-based sensor was 1.5, 2, and 2.5 times greater than that of the woven CCF-based sensor, Figure S2: Relationship between the compression distance and applied pressure of knitted and woven carbonized cotton fabrics, Table S1: Variances of the capacitance variations of 5-s releasing and 5-s grasping motions with a $25 \%, 50 \%$, $75 \%$, and $100 \%$ filled water cup.

Author Contributions: Conceptualization, Y.K. and J.K.; methodology, Y.K., C.C.V., and J.K.; validation, Y.K. and J.K.; formal analysis, Y.K.; investigation, Y.K.; prototype fabrication, Y.K. and C.C.V.; data curation, Y.K. and C.C.V.; writing-original draft preparation, Y.K.; writing-review and editing, Y.K., C.C.V., and J.K.; visualization, Y.K.; supervision, J.K.; project administration, J.K.; funding acquisition, J.K. All authors have read and agreed to the published version of the manuscript.

Funding: This research was partly supported by a National Research Foundation of Korea (NRF2019R1A2C2005933) grant funded by the Korea Government (MSIT) and a Korea Institute for Advancement of Technology (KIAT) grant funded by the Korea Government (MOTIE) (P0002397, HRD program for the Industrial Convergence of Wearable Smart Devices).

Institutional Review Board Statement: Not applicable.

Informed Consent Statement: Not applicable.

Data Availability Statement: Not applicable.

Acknowledgments: We thank Bomi Kim for providing administrative support.

Conflicts of Interest: The authors declare no conflict of interest.

\section{References}

1. Hwang, J.; Kim, Y.; Yang, H.; Oh, J.H. Fabrication of hierarchically porous structured PDMS composites and their application as a flexible capacitive pressure sensor. Compos. B Eng. 2021, 211, 108607. [CrossRef]

2. Wu, R.; Ma, L.; Patil, A.; Meng, Z.; Liu, S.; Hou, C.; Zhang, Y.; Yu, W.; Guo, W.; Liu, X.Y. Graphene decorated carbonized cellulose fabric for physiological signal monitoring and energy harvesting. J. Mater. Chem. A 2020, 8, 12665-12673. [CrossRef]

3. Jung, Y.; Lee, W.; Jung, K.; Park, B.; Park, J.; Ko, J.; Cho, H. A highly sensitive and flexible capacitive pressure sensor based on a porous three-dimensional PDMS/microsphere composite. Polymers 2020, 12, 1412. [CrossRef]

4. Takamatsu, S.; Lonjaret, T.; Ismailova, E.; Masuda, A.; Itoh, T.; Malliaras, G.G. Wearable keyboard using conducting polymer electrodes on textiles. Adv. Mater. 2016, 28, 4485-4488. [CrossRef] [PubMed]

5. Guo, Y.; Guo, Z.; Zhong, M.; Wan, P.; Zhang, W.; Zhang, L.J.S. A flexible wearable pressure sensor with bioinspired microcrack and interlocking for full-range human-machine interfacing. Small 2018, 14, 1803018. [CrossRef] [PubMed]

6. Fastier-Wooller, J.; Dinh, T.; Dau, V.T.; Phan, H.-P.; Yang, F.; Dao, D.V. Low-cost graphite on paper pressure sensor for a robot gripper with a trivial fabrication process. Sensors 2018, 18, 3300. [CrossRef] [PubMed]

7. Sekine, T.; Abe, M.; Muraki, K.; Tachibana, S.; Wang, Y.-F.; Hong, J.; Takeda, Y.; Kumaki, D.; Tokito, S. Microporous Induced Fully Printed Pressure Sensor for Wearable Soft Robotics Machine Interfaces. Adv. Intell. Syst. 2020, 2, 2000179. [CrossRef]

8. Zhu, L.; Wang, Y.; Mei, D.; Jiang, C.J.M. Development of Fully Flexible Tactile Pressure Sensor with Bilayer Interlaced Bumps for Robotic Grasping Applications. Micromachines 2020, 11, 770. [CrossRef] 
9. Liu, W.; Liu, N.; Yue, Y.; Rao, J.; Cheng, F.; Su, J.; Liu, Z.; Gao, Y. Piezoresistive pressure sensor based on synergistical innerconnect polyvinyl alcohol nanowires/wrinkled graphene film. Small 2018, 14, 1704149. [CrossRef]

10. Zhao, T.; Li, T.; Chen, L.; Yuan, L.; Li, X.; Zhang, J. Highly sensitive flexible piezoresistive pressure sensor developed using biomimetically textured porous materials. ACS Appl. Mater. Interfaces 2019, 11, 29466-29473. [CrossRef]

11. Atalay, O.; Atalay, A.; Gafford, J.; Walsh, C. A highly sensitive capacitive-based soft pressure sensor based on a conductive fabric and a microporous dielectric layer. Adv. Mater. Technol. 2018, 3, 1700237. [CrossRef]

12. Wen, Z.; Yang, J.; Ding, H.; Zhang, W.; Wu, D.; Xu, J.; Shi, Z.; Xu, T.; Tian, Y.; Li, X. Ultra-highly sensitive, low hysteretic and flexible pressure sensor based on porous MWCNTs/Ecoflex elastomer composites. J. Mater. Sci. Mater. Electron. 2018, 29, 20978-20983. [CrossRef]

13. Park, S.W.; Das, P.S.; Chhetry, A.; Park, J.Y. A flexible capacitive pressure sensor for wearable respiration monitoring system. IEEE Sens. J. 2017, 17, 6558-6564. [CrossRef]

14. Yoon, J.I.; Choi, K.S.; Chang, S.P. A novel means of fabricating microporous structures for the dielectric layers of capacitive pressure sensor. Microelectron. Eng. 2017, 179, 60-66. [CrossRef]

15. Yang, Y.; Pan, H.; Xie, G.; Jiang, Y.; Chen, C.; Su, Y.; Wang, Y.; Tai, H. Flexible piezoelectric pressure sensor based on polydopaminemodified $\mathrm{BaTiO}_{3} / \mathrm{PVDF}$ composite film for human motion monitoring. Sens. Actuator A Phys. 2020, 301, 111789. [CrossRef]

16. Hosseini, E.S.; Manjakkal, L.; Shakthivel, D.; Dahiya, R. Glycine-chitosan-based flexible biodegradable piezoelectric pressure sensor. ACS Appl. Mater. Interfaces 2020, 12, 9008-9016. [CrossRef]

17. Wang, X.; Zhang, H.; Dong, L.; Han, X.; Du, W.; Zhai, J.; Pan, C.; Wang, Z.L. Self-powered high-resolution and pressure-sensitive triboelectric sensor matrix for real-time tactile mapping. Adv. Mater. 2016, 28, 2896-2903. [CrossRef] [PubMed]

18. Yang, J.; Luo, S.; Zhou, X.; Li, J.; Fu, J.; Yang, W.; Wei, D. Flexible, tunable, and ultrasensitive capacitive pressure sensor with microconformal graphene electrodes. ACS Appl. Mater. Interfaces 2019, 11, 14997-15006. [CrossRef]

19. Ma, L.; Shuai, X.; Hu, Y.; Liang, X.; Zhu, P.; Sun, R.; Wong, C.-P. A highly sensitive and flexible capacitive pressure sensor based on a micro-arrayed polydimethylsiloxane dielectric layer. J. Mater. Chem. C 2018, 6, 13232-13240. [CrossRef]

20. Zang, Y.; Zhang, F.; Di, C.-A.; Zhu, D. Advances of flexible pressure sensors toward artificial intelligence and health care applications. Mater. Horiz. 2015, 2, 140-156. [CrossRef]

21. Xiong, Y.; Shen, Y.; Tian, L.; Hu, Y.; Zhu, P.; Sun, R.; Wong, C.-P. A flexible, ultra-highly sensitive and stable capacitive pressure sensor with convex microarrays for motion and health monitoring. Nano Energy 2020, 70, 104436. [CrossRef]

22. Ruth, S.R.A.; Beker, L.; Tran, H.; Feig, V.R.; Matsuhisa, N.; Bao, Z. Rational design of capacitive pressure sensors based on pyramidal microstructures for specialized monitoring of biosignals. Adv. Funct. Mater. 2020, 30, 1903100. [CrossRef]

23. Luo, Y.; Shao, J.; Chen, S.; Chen, X.; Tian, H.; Li, X.; Wang, L.; Wang, D.; Lu, B. Flexible capacitive pressure sensor enhanced by tilted micropillar arrays. ACS Appl. Mater. Interfaces 2019, 11, 17796-17803. [CrossRef] [PubMed]

24. Shuai, X.; Zhu, P.; Zeng, W.; Hu, Y.; Liang, X.; Zhang, Y.; Sun, R.; Wong, C.-P. Highly sensitive flexible pressure sensor based on silver nanowires-embedded polydimethylsiloxane electrode with microarray structure. ACS Appl. Mater. Interfaces 2017, 9 , 26314-26324. [CrossRef] [PubMed]

25. Choi, J.; Kwon, D.; Kim, K.; Park, J.; Orbe, D.D.; Gu, J.; Ahn, J.; Cho, I.; Jeong, Y.; Oh, Y. Synergetic effect of porous elastomer and percolation of carbon nanotube filler toward high performance capacitive pressure sensors. ACS Appl. Mater. Interfaces 2019, 12, 1698-1706. [CrossRef]

26. Kwon, D.; Lee, T.-I.; Shim, J.; Ryu, S.; Kim, M.S.; Kim, S.; Kim, T.-S.; Park, I. Highly sensitive, flexible, and wearable pressure sensor based on a giant piezocapacitive effect of three-dimensional microporous elastomeric dielectric layer. ACS Appl. Mater. Interfaces 2016, 8, 16922-16931. [CrossRef]

27. Long, Y.; Zhao, X.; Jiang, X.; Zhang, L.; Zhang, H.; Liu, Y.; Zhu, H. A porous graphene/polydimethylsiloxane composite by chemical foaming for simultaneous tensile and compressive strain sensing. Flatchem 2018, 10, 1-7. [CrossRef]

28. Kim, Y.; Jang, S.; Oh, J.H. Fabrication of highly sensitive capacitive pressure sensors with porous PDMS dielectric layer via microwave treatment. Microelectron. Eng. 2019, 215, 111002. [CrossRef]

29. Mannsfeld, S.C.; Tee, B.C.; Stoltenberg, R.M.; Chen, C.V.H.; Barman, S.; Muir, B.V.; Sokolov, A.N.; Reese, C.; Bao, Z. Highly sensitive flexible pressure sensors with microstructured rubber dielectric layers. Nat. Mater. 2010, 9, 859-864. [CrossRef]

30. Han, M.; Lee, J.; Kim, J.K.; An, H.K.; Kang, S.-W.; Jung, D. Highly sensitive and flexible wearable pressure sensor with dielectric elastomer and carbon nanotube electrodes. Sens. Actuator A Phys. 2020, 305, 111941. [CrossRef]

31. Li, W.; Jin, X.; Zheng, Y.; Chang, X.; Wang, W.; Lin, T.; Zheng, F.; Onyilagha, O.; Zhu, Z. A porous and air gap elastomeric dielectric layer for wearable capacitive pressure sensor with high sensitivity and a wide detection range. J. Mater. Chem. C 2020, 8, 11468-11476. [CrossRef]

32. Chang, S.; Li, J.; He, Y.; Liu, H.; Cheng, B. A high-sensitivity and low-hysteresis flexible pressure sensor based on carbonized cotton fabric. Sens. Actuator A Phys. 2019, 294, 45-53. [CrossRef]

33. Sun, C.; Li, X.; Cai, Z.; Ge, F. Carbonized cotton fabric in-situ electrodeposition polypyrrole as high-performance flexible electrode for wearable supercapacitor. Electrochim. Acta 2019, 296, 617-626. [CrossRef]

34. Zhang, M.; Wang, C.; Wang, H.; Jian, M.; Hao, X.; Zhang, Y. Carbonized cotton fabric for high-performance wearable strain sensors. Adv. Funct. Mater. 2017, 27, 1604795. [CrossRef]

35. Deng, C.; Pan, L.; Cui, R.; Li, C.; Qin, J. Wearable strain sensor made of carbonized cotton cloth. J. Mater. Sci. Mater. Electron. 2017, 28, 3535-3541. [CrossRef] 
36. Zhang, L.; Li, H.; Lai, X.; Gao, T.; Liao, X.; Chen, W.; Zeng, X. Carbonized cotton fabric-based multilayer piezoresistive pressure sensors. Cellulose 2019, 26, 5001-5014. [CrossRef]

37. Chang, S.; Li, J.; He, Y.; Liu, H. Effects of carbonization temperature and substrate concentration on the sensing performance of flexible pressure sensor. J. Appl. Phys. 2020, 126, 1-10. [CrossRef]

38. Eom, S.; Lim, S. Stretchable complementary split ring resonator (CSRR)-based radio frequency (RF) sensor for strain direction and level detection. Sensors 2016, 16, 1667. [CrossRef] [PubMed]

39. Chen, S.; Zhuo, B.; Guo, X. Large area one-step facile processing of microstructured elastomeric dielectric film for high sensitivity and durable sensing over wide pressure range. ACS Appl. Mater. Interfaces 2016, 8, 20364-20370. [CrossRef]

40. Yoon, S.G.; Koo, H.-J.; Chang, S.T. Highly stretchable and transparent microfluidic strain sensors for monitoring human body motions. ACS Appl. Mater. Interfaces 2015, 7, 27562-27570. [CrossRef]

41. Nguyen, S.-T. Effect of pore shape on the effective behavior of viscoelastic porous media. Int. J. Solids Struct. 2017, 125, 161-171. [CrossRef] 